\title{
escrito pornn.
}

\section{'Irvyn E. Nieves-Rolón}

Catedrático Asociado en el Departamento de Trabajo Social, Universidad Ana G. Méndez, Recinto de Carolina.

$\triangle$ inieves12@suagm.edu

(D) ORCID ID https://orcid.org/0000-0002-4218-0316

\section{Eileen Rivera Rodríguez}

Trabajadora Social Clínica de la Corporación de Servicios Médicos en Arecibo, Puerto Rico.

\eileenrivera47@yahoo.com

(D) ORCID ID https://orcid.org/0000-0001-5905-1580

\section{${ }^{3}$ Verónica C. Ortiz-Candelaria}

Estudiante graduada de Trabajo Social Clínico, Universidad Ana G. Méndez, Recinto de Carolina.

$\triangle$ veronica.cristinaortiz@gmail.com

(D) ORCID ID https://orcid.org/0000-0001-5304-2181

\section{Krystal L. Pérez-Martínez}

Estudiante graduada de Trabajo Social Clínico, Universidad Ana G. Méndez, Recinto de Carolina.

\kperez152@email.suagm.edu

(D) ORCID ID https://orcid.org/0000-0002-6442-6858

Cómo citar / citation:

Nieves-Rolón, I., Rivera, E., Ortiz-Candelaria, V., \& Pérez-Martínez, K. (2019).

Trabajo Social Clínico en Puerto Rico: Alcances y debates que rodean su práctica. Voces desde el Trabajo Social, 7(1), 14-35. https://doi.og/10.31919/voces.v7i1.73

Recibido / received: 12 de abril de 2019
Revisado / reviewed: 3 de julio de 2019
Aceptado / accepted: 10 de octubre de 2019

Derechos de autoría / Copyright: (c) 2019 Nieves-Rolón, I. et al. Este es un artículo de acceso abierto y distribuido bajo los términos de la licencia y políticas de Creative Commons Attribution 4.0 International License. 


\section{TRABAJO SOCIAL CLÍNICO EN PUERTO RICO: ALCANCES Y DEBATES QUE RODEAN SU PRÁCTICA}

Irvyn E. Nieves-Rolón', Eileen Z. Rivera Rodríguez², (D) Verónica C. Ortiz-Candelaria ${ }^{3}$, Krystal L. Pérez-Martínez ${ }^{4}$

\section{Resumen}

G openaccess PeEr-Reviewed

El presente trabajo discute hallazgos de una investigación en la que se profundiza sobre los alcances del Trabajo Social Clínico en Puerto Rico. Para este estudio de diseño cualitativo y narrativo, se realizaron entrevistas a profesionales del Trabajo Social Clínico que, además de un grado de maestría en Trabajo Social, cuentan con un grado doctoral en Psicología. En Puerto Rico, se reconoce la aportación de esta especialidad, particularmente, en los escenarios de intervención directa. Se documenta que el Trabajo Social con especialidad clínica, continúa siendo eje de discusión. Asuntos como la definición de la especialidad y los alcances de la misma, quedan sujetos a las opiniones o posicionamientos paradigmáticos desde los adentros de la propia profesión. El escrito presenta las perspectivas de las personas entrevistadas sobre la relación y las diferencias entre el Trabajo Social Clínico y otras profesiones. Se señala la relevancia de la formación académica y se reflexiona sobre tensiones y posturas que deberían ser atendidas por los profesionales. Por último, se realizan recomendaciones priorizando la importancia de abrir canales de diálogo para dilucidar debates existentes con relación a la especialidad y a la profesión del Trabajo Social en general. 


\section{Palabras claves}

Trabajo Social Clínico, Dimensión Terapéutica del Trabajo Social, Especialidad en Trabajo Social, Educación en Trabajo Social, Intervención Social

\section{Sumario}

Introducción. Método de la investigación. Resultados. Discusión de hallazgos. Referencias.

\section{[EN] CLINICAL SOCIAL WORK IN PUERTO RICO: EXTENTS AND DEBATES SURROUNDING ITS PRACTICE}

\section{Abstract}

This paper discusses the findings of a research that delves into the extents of Clinical Social Work in Puerto Rico. For this qualitative and narrative design study, interviews were conducted with Clinical Social Work professionals who, in addition to a master's degree in Social Work, have a doctoral degree in Psychology. In Puerto Rico, the contribution of this clinical specialization is recognized, particularly in the direct intervention settings. It is documented that Social Work with a clinical specialization continues to be the axis of discussions. Issues such as its definition and scope are subject to the opinions or paradigmatic positions from within the profession itself. The paper presents the perspectives of the interviewed professionals about the relationship and differences between Clinical Social Work and other professions. The relevance of the academic training is pointed out, as well as reflections of the tensions and stances that should be attended by the profession. Finally, recommendations are made prioritizing the importance of opening channels of dialogue to elucidate existing debates in relation to the specialization and the profession of Social Work in general.

\section{Keywords}

Clinical Social Work, Therapeutic Dimension of Social Work, Social Work Specialization, Social Work Education, Social Intervention. 


\section{Introducción}

La práctica del Trabajo Social Clínico (TSC) ha ido desarrollándose en Puerto Rico, a su vez, ha ido estableciéndose la especialidad en la Academia. Al presente, varias universidades en Puerto Rico ofrecen dicha especialidad. Sin embargo, se observan diferencias en cuanto a las formas de entender el TSC.

Por la forma en que se ha concebido el Trabajo Social (TS) en Puerto Rico, la práctica clínica se exhibe de manera distinta a los Estados Unidos y otros países en donde la especialidad está más organizada. Por ejemplo, en algunos lugares requiere, una licencia profesional especializada (ABECSW, 2019). Entonces, es posible encontrar concepciones muy diversas sobre los roles y funciones del TSC, particularmente cuando se compara con otras profesiones.

La Asociación Nacional de Trabajadores Sociales, por sus siglas en inglés NASW (2019), explica que el TSC se enfoca en la evaluación, diagnóstico, tratamiento y prevención de enfermedades mentales, emocionales y disturbios conductuales. Desde esta postura, entran al debate aquellos servicios para los cuales el TSC está capacitado. Aspectos como evaluación, diagnóstico, tratamiento -incluyendo psicoterapia y consejería-, quedan al borde de los debates y diversos posicionamientos de quienes ejercen la profesión.
Asimismo, algunos plantean que hacen más de 50 años diversos autores han defendido que: "la práctica clínica con personas y familias es parte de la esencia constitutiva del TS, reconociendo nexos con corrientes psicoterapéuticas, psicosociales y psiquiátricas" (Huaiquiche \& Bastías, 2016, p.34). Por otro lado, otros autores plantean que es necesario mirar el entorno sociopolítico en que se potencia el TSC. Circunstancias como la precarización de las políticas sociales, el encarecimiento y desplazamiento de servicios sociales hacia el sector privado y la psicologización de problemas socioestructurales han llevado a un aumento considerable de profesionales que se especializan en TSC (Barreto, Rosario y Sánchez, 2016).

El TSC, como toda la profesión del TS, procura mejorar y mantener el funcionamiento psicosocial de los individuos, familias y grupos (Córdova, 2010). No obstante, existen otras profesiones clínicas como la psicología, psiquiatría, y la enfermería psiquiátrica, con quienes se pueden encontrar oficios coincidentes. Aspectos como las áreas de dominio o incluso sobre la propia definición del TSC y su relación con las bases de la profesión puede ser tema de debate. Este escrito procura aportar a la discusión de tales aspectos.

La realización de esta investigación siguió una serie de objetivos que 
sobrepasan lo que puede presentarse en este artículo debido a razones de espacio. Los resultados que se presentan corresponden a la búsqueda por responder las siguientes preguntas de investigación: (1) ¿Cómo definen el trabajo social clínico quienes ejercen la profesión?; y (2) ¿Cuáles aspectos del ejercicio del trabajo social clínico son materia de debate en Puerto Rico?

\section{Método de la investigación}

\section{Diseño}

Este estudio sigue un diseño cualitativo de investigación. La investigación cualitativa procura recopilar información sin tener por base la medición numérica mientras, viabiliza reconstruir la realidad tal como la observan los actores (Hernández, Fernández \& Baptista, 2014). Los estudios cualitativos enfatizan los significados subjetivos mientras asumen que la realidad es dinámica (Padgett, 2008). Esto atañe a esta investigación dada las circunstancias en que el TSC es entendido de diversas formas y con diferentes alcances.

Este estudio es de tipo exploratorio y narrativo debido a que el objetivo consiste en estudiar un tema poco abordado. Los estudios narrativos permiten tener acceso a las perspectivas y experiencias para describirlas y analizarlas (Elçi \& Devran, 2014; Hernández et al.,
2014). Esto resulta muy pertinente a esta investigación ya que recoge las perspectivas de los propios sujetos que ejercen la profesión.

Estrategias de recopilación de información

Para la recopilación de información se realizaron 7 entrevistas semi estructuradas. Vale establecer que la entrevista cualitativa resulta ideal para los propósitos de este estudio. La misma permite la recolección de información detallada sobre un tema específico, además, de ser una técnica ágil para recolectar información sobre experiencias, creencias y conductas (Babbie \& Rubin, 2011; Ryan, Coughlan, \& Cronin, 2009).

\section{Participantes}

Debido al tema y los debates previstos en la realización de la investigación se consideró conveniente que los participantes del estudio tuvieran una formación mixta en trabajo social y psicología. De esta forma, los criterios de inclusión fueron: (1) haber ejercido 2 años o más como trabajador social clínico, ofreciendo servicio directo en una organización pública o privada en Puerto Rico; y (2) poseer un grado de maestría en trabajo social y un doctorado en psicología. Esta particularidad facilitó que, al entrar en la discusión, los participantes pudieran compartir sus percepciones desde los adentros de la 
propia dualidad que significan las dos profesiones en las cuales se formaron. Es sumamente importante destacar que la obtención del grado doctoral en psicología no inhabilita la formación y experiencias obtenidas por los participantes como profesionales del trabajo social, profesión para la cual, además de ejercerla, alcanzaron grados de maestría. Muy por el contrario, lo que se procura es que participantes que han experimentado los saberes y procederes de cada profesión, puedan articular sus diferencias y similitudes.

Para el reclutamiento de los 7 participantes que son profesionales del TSC y, a su vez, doctores en psicología, se utilizó la técnica de muestreo conocida como "bola de nieve". Dicha técnica consiste en notificar a alguna persona con las características que persigue la investigación y que ésta, a su vez, notifique a otros sujetos con similares características (Ellard-Gray, Choubak \& Crann, 2015). Este tipo de muestreo se utiliza cuando no existe abundancia de personas con dichas características y se entiende que sería más viable conseguir su participación por referido de personas del mismo grupo (Padgett, 2008).

\section{Características de los participantes}

Participaron 7 profesionales. Cuatro (4) se identificaron como féminas y tres (3) de género masculino. La totalidad de las personas entrevistadas realizaron sus estudios graduados tanto de maestría en TS, como de doctorado en Psicología en universidades localizadas en Puerto Rico. Dos (2) participantes realizaron su maestría en TS en la Pontificia Universidad Católica, tres (3) en la Universidad de Puerto Rico y dos (2) en la Universidad Interamericana. Los grados doctorales en psicología los obtuvieron, tres (3) participantes en la Pontificia Universidad Católica, dos (2) en la Universidad Carlos Albizu, uno (1) en la Universidad de Puerto Rico y uno (1) en la Universidad Interamericana. Es importante destacar que la diversidad de Instituciones académicas en las cuales se formaron, particularmente por ser universidades en Puerto Rico, añade valor a la discusión. No obstante, dicha característica incidental, no tiene presunción de representatividad alguna, dado el método cualitativo empleado en este estudio.

Procedimiento para el análisis de la información

Para las entrevistas se utilizaron guiones con preguntas. Todas las entrevistas fueron audio-grabadas, con la autorización de las personas participantes. Luego de realizada cada entrevista fueron transcritas 'ad verbatim' y analizadas. En el proceso de análisis de todas las entrevistas, se fueron identificando categorías emergentes. Estas categorizaciones permitieron la organización de las narrativas que a continuación se 
presentan. Debido a las características del tema abordado en este estudio se utilizó el "Análisis de discurso" como tipo de análisis cualitativo. Se trata de descifrar el sentido implícito de las expresiones analizadas (Sayago, 2014). El análisis de discurso permite identificarcómolos diferentes discursos dan estructura a la forma en que se producen significados, identidades y otras realidades sociales.

\section{Consideraciones éticas}

Al realizar procesos de investigación social es imprescindible realizar esfuerzos dirigidos a proteger los sujetos humanos que participan del estudio. Con tal propósito esta investigación fue sometida y aprobada por la Junta para la Protección de Sujetos Humanos en la Investigación, del Sistema Universitario Ana G. Méndez. El número de protocolo asignado a la investigación fue 02-054-14.

\section{Resultados}

La presentación de resultados a continuación es producto del análisis realizado a las entrevistas. La discusión se establece hilvanando, de forma literal, o bien sea 'ad verbatim', las verbalizaciones de las personas entrevistadas. Esta ilación de expresiones se hizo a partir de las categorías que emergieron en el proceso de análisis. Mientras se presentan las verbalizaciones, se hacen comentarios y observaciones para contextualizar lo presentado. Debido a que se guarda la confidencialidad, las personas entrevistadas fueron identificadas con un número que varió entre el 01 y el 07 . Las verbalizaciones se presentan con dicho número identificador.

Uno de los primeros aspectos que surgen de las entrevistas, es la propia definición del TSC. Al momento de estructurar lo que define al TSC se presentan distintas consideraciones y no se observa una definición homogénea: "ahí es que está el debate principal, que todos vemos el Trabajo Social Clínico desde una manera, desde una perspectiva distinta y no nos ponemos de acuerdo en lo que realmente es la definición del Trabajo Social Clínico" (01); "dentro de la misma profesión de Trabajo Social hay unas cosas que son puntos grises, las cuales no están definidas" (02); "...para unas personas ser clínico es estar en un escenario de salud mental haciendo de referir gente, de dar, este, grupos psicoeducativos y cosas como esa. Para otros es dar terapia" (07).

No obstante, hay varias expresiones que llevan a enfatizar que las bases del TSC permanecen en la identidad del TS: "...nosotros tenemos unas bases que son comunitarias, que son prácticamente de individuos, de factores ambientales y de comunidad" (05); 
...el Trabajo Social no se puede divorciar de sus aspectos fundamentales y de su base. Y la base es este proceso, esta base de solución de problemas. Este proceso generalista de lo que es el Trabajo Social. Porque si no, se va a desvirtuar. No puede verse como una práctica clínica nada más (03).

De forma general se observó dificultad al momento de definir concretamenteal TSC. En ocasiones la definición provista resultaba imprecisa o indiferenciable de otras áreas del TS. Por ejemplo: “... el Trabajo Social Clínico es, eh, la profesión que se dedica a trabajar el aspecto biopsicosocial del individuo, en todas sus manifestaciones, individuo, grupo, familia y comunidad" (01);

...el Trabajo Social Clínico es, es un campo profesional de intervención que va dirigido a, a manejar cualquier tipo de población [...] utilizando unos marcos teóricos propios de la profesión basándose en teorías eh, que están elaboradas relacionadas al, al comportamiento humano (04).

Se observa que mientras algunos profesionales ofrecen definiciones poco precisas, otros destacan en sus narrativas conceptos como 'biopsicosocial', 'sistémico' y 'bases comunitarias'. Otras narrativas, al definir TSC, presentan conceptos como 'psicopatología de un individuo' y 'psiquis':

\section{... (el TSC) es un tipo de Trabajo}

Social como especialidad donde se dirige a trabajar aspectos que tienen que ver con la psicopatología de un individuo. No solamente cubre el aspecto social, del entorno social, verdad lo macro, lo mezzo, sino que también, esto, cubre la parte micro viendo al individuo, verdad, como también un ente en el cual la psiquis eh, se ve afectada (02).

Otra forma de acercarse a definir el TSC, es por medio de las áreas de dominio. Los profesionales entrevistados hacen hincapié en el conocimiento y manejo del Manual Diagnóstico y Estadístico de los Trastornos Mentales (DSM). Asimismo, dominio en el conocimiento y aplicación de modelos que permiten trabajar con las patologías identificadas en los sujetos, como algo esencial para lograr demostrar las competencias necesarias de un profesional clínico en salud mental. El participante 02, plantea:

... (un TSC debe tener) dominio pleno en bases teóricas. Dominio pleno en lo que son signos y síntomas de patologías [...] Si no hay dominio de teorías, si no hay 
dominio de patologías se va a carecer, verdad, de [...] hacer una identificación de qué es lo que le pasa al cliente.

Otros participantes aducen: “... debe conocer la sintomatología eh, que se identifique con los diagnósticos verdad, más comunes [...] como son la ansiedad, la depresión, trastorno bipolar, este, las fobias [...] conocer eh, el DSM-V" (06);

...dominio absoluto de lo, del instrumento que se utiliza para el diagnóstico, que es el DSM$\mathrm{V}$, cuando digo completo es de la $A$ a la Z. Si carece de ese adiestramiento, verdad, entonces va a tener, esto, mucha dificultad para hacer un, un diagnóstico como tal (02).

En relación a la capacidad del TSC para realizar diagnósticos de salud mental, se presenta un debate interesante. En el proceso de entrevista surgió la perspectiva de que los profesionales del TSC, no pueden realizar diagnósticos y, en su lugar, realizan impresiones diagnósticas. participante 02, argumenta:

Dentro de la formación verdad, que yo he tenido [...] pues soy Trabajador Social, eh y además, soy Psicólogo Clínico, [...] siempre se me enseñó (que) el Psicólogo es el que diagnostica y el Trabajador Social el que da una impresión diagnóstica. [...] Entonces, cuando yo digo que el Trabajador Social da impresión diagnóstica, es que puede dar impresión de los trastornos generales, más comunes, pero cuando hablamos de, de trastornos [...] hay una cantidad de trastornos, innumerables, que no necesariamente el Trabajador Social Clínico tiene el adiestramiento para cada uno de estos trastornos (02).

El participante 03, se distancia de dicha postura y arguye:

Claro que diagnostica. Siempre eh, debe diagnosticar. Para eso se hace, para eso existe el Trabajo Social Clínico, para poder diagnosticar. Para eso se prepara en el DSM [...] en la psicopatología. Porque si, entonces, [...] 'no, lo que hace el Trabajador Social es una impresión diagnostica'... y ¿Qué es una impresión diagnostica?, ¿Qué es un diagnóstico? Todos tenemos que hacer una impresión diagnóstica, tenemos que identificar, esa es la impresión diagnóstica (03).

Sin lugar a duda es meritorio continuar dialogando sobre aspectos de debate, como el diagnóstico, y la amplitud de argumentos que pueden circunvenir su análisis. 
En una consideración similar, otros participantes establecen que hay áreas fuera de las competencias del TSC y que las mismas corresponden a otras disciplinas clínicas: "los trastornos de personalidad, mayormente, les competeaellos(psicólogos). Trabajarlos no nos compete a nosotros” (05); “...en esa área específica (farmacoterapia), en ese tipo de consejería nosotros no debemos entrar [...] y esa no es nuestra área de expertise, nada que tenga que ver con farmacoterapia debemos entrar (06)";

la Psicología Clínica que tiene unos instrumentos estandarizados eh, eh, que se utilizan para medir el funcionamiento de la persona como, por ejemplo, algo tan básico como es el cociente inte, intelectual. Obviamente el Trabajador Social no, no, no tiene las herramientas para utilizar ese proceso (04).

Otro aspecto que emergió en la discusión, y que sin lugar a duda tiene gran relevancia, es la formación que se ofrece en los programas de TSC en las universidades localizadas en Puerto Rico. Sería lógico pensar, al menos deseable, que la formación provista en las universidades debe encausar esas características que hacen particular al TSC. Sin embargo, las verbalizaciones de los participantes de la investigación no se dirigen a la identificación de esos aspectos. Resulta ser una coincidencia interesante, que aspectos que han sido identificados previamente por los participantes como "áreas de dominio" del TSC, diagnóstico, psicopatologías, teorías y modelos de intervención, son también identificadas como las "áreas a fortalecer" al presente, en la formación de profesionales del TS con especialidad clínica. Al respecto, el participante 01 dice: “...fortalecería el programa precisamente en el área clínica que tiene que ver con las teorías y modelos, verdad, de, de intervención clínica, (ofrecer) más de un curso de psicopatología"; "... hay que trabajar con lo que es áreas de especialización, fortalecer las áreas eh, seguir fortaleciendo las áreas de diagnóstico eh, teorías eh, planes de tratamiento" (02).

Hay que reconocer las interpretaciones que pueden estar en la base de esta línea argumentativa. Puede ser el caso que esto es planteado con el ánimo de señalar que se debe mejorar aspectos que son propios del TSC y deben seguir desarrollándose como cualquier otro aspecto que un profesional procura mejorar en sus áreas de desempeño; o se reconoce la contradicción de que, aunque se alegue esas áreas de dominio del TSC, realmente no lo son en la práctica.

Por otra parte, a pesar de las dificultades para definir al TSC, entre 
los profesionales se identificó que existe cierta uniformidad al momento de establecer las diferencias con la psicología clínica. Los profesionales consideran que su enfoque como TSC está dirigido a manejar estresores ambientales, mientras que la psicología clínica se enfoca en aspectos intrapsíquicos. Por ejemplo: “... el Trabajador Social Clínico recopila información de la comunidad o del recurso de apoyo del cliente que el Psicólogo, generalmente, eso no lo hace" (04); "(al TSC) compete trabajar la estabilización, minimizar síntomas y riesgos y trabajar con los aspectos ambientales que influyen a que (el participante) no siga recayendo en múltiples hospitalizaciones" (05). Por su parte, el participante 01 dice:

... (el TSC) es un poquito más holístico porque toma en consideración el aspecto social, del individuo y el Psicólogo Clínico se enfoca más en la psiquis, de los, en los trastornos que en los problemas emocionales de la gente. Verdad, así que hay una diferencia en estos dos, este, en esas dos profesiones.

Mientras que el participante 04 dice:

...A diferencia, quizás, de la

Psicología Clínica [...] que va más focalizado a ver al sujeto desde el punto de vista más intra, más que de afuera; verdad, no, no le da tanto énfasis a los elementos externos, verdad, y cómo el ambiente puede influenciar la situación en el funcionamiento del individuo. Eso es, básicamente, la, la diferencia principal que yo entiendo que eh, hay entre Trabajo Social Clínico versus Psicología, por darte un ejemplo."

Sin embargo, a pesar de que establecen una diferenciación clara del enfoque entre disciplinas, los profesionales entrevistados entienden que la práctica del TSC está basada en teorías y modelos producto de la investigación en el campo de la psicología clínica. Aunque destacan que su uso es distinto y se identifican en las narrativas conceptos como 'sistémico' y 'holístico', para algunos esto no representa un dilema epistémico: “... utilizamos las mismas teorías, pero la visión que utilizamos para acercarnos al, al sujeto, al cliente eh, es diverso y generalmente está basado en, en, en un enfoque sistémico" (04). No obstante, para otros, esto representa un punto de debate debido a que consideran contradictorio que una profesión que resalta el aspecto holístico de su intervención utilice modelos y teorías que no respondan a estas bases: 
... Igualmente, otro debate que puede surgir en la profesión que para mí es bien importante, es que el Trabajo Social le toma prestado mucho los modelos a otras profesiones como es la Psicología Clínica. Porque los Psicólogos Clínicos se han dado a la tarea de investigar, de crear unos modelos de tratamiento, unas teorías para tratar las condiciones y no necesariamente desde unas perspectivas de, de, de, de, holística como es el Trabajador Social Clínico. Y nosotros dependemos de esos modelos y esas teorías para, precisamente hacer el Trabajo Social Clínico (01).

Interesantemente, otro aspecto identificado como área a fortalecer en el TSC, es el dominio de las destrezas de investigación: "lo que es el campo investigativo [...] en la dimensión que sea, verdad, debería de fortalecerla y buscar un área de especialidad" (02); "procesos de investigación [...] yo creo que eso es otro elemento que, que hay, que hay que incluir" (04). Este particular se destaca sumado al reconocimiento de la necesidad de que se generen marcos teóricos y procesos de intervención concebidos desde el TS y no tomados de la psicología. Definitivamente, incentivar y fortalecer las destrezas de investigación en los profesionales del TSC, podrían conducir a que se desarrollen teorías y modelos de intervención que correspondan con mayor adecuación a las cualidades particulares del TSC.

Otra vertiente que se identifica en las narrativas y que aporta a las dificultades para definir concretamente la práctica del TSC, es la situación de identidad política de Puerto Rico. Concretamente, el conflicto de identidad del TS en Puerto Rico, tal como sucede con la sociedad puertorriqueña en general. Por una parte, se prioriza la relación con los Estados Unidos de América y, por otra, se atesora y defiende la identidad latinoamericana. Teniendo en ocasiones el efecto, de depender de definiciones del TS, en este caso TSC, que resultan más heterónomas que autónomas:

...en lo que es el gremio del

Trabajo Social hay unas corrientes muy difíciles, políticas diría yo, verdad, entre Latinoamérica, sur América [...] Estados Unidos eh, la mirada anglosajón. Nosotros emulamos, emulamos un Trabajo Social Clínico que viene de Estados Unidos, verdad y por nuestra posición política hay unas corrientes eh, que, que en, en sur América pues el Trabajo Social tiene otra visión por, por sus posturas políticas [...]. Entonces aquí, aquí como siempre estamos 
mirando a diferentes áreas y nunca podemos ponernos de acuerdo con nosotros y definirlo desde nuestro proceso de, de ser puertorriqueños y el impacto que tiene este, de diferentes vertientes, consolidarlo (03).

Este particular es una situación que divide la población puertorriqueña. Seguramente, no sería una sorpresa constatar que las mismas posturas que dividen a la sociedad en torno a asuntos de política partidista 0 estatutaria, laceren la forma en que profesionales miran e interpretan los alcances y debates del TSC. Aunque, ciertamente, hay muchas variables a considerar a la hora de entender las formas de enfrentar estos debates, una de ellas es la intolerancia para lidiar con las posturas contrarias, junto a la actitud de rehusarse a considerar y re-valorar los posicionamientos propios.

\section{Discusión de hallazgos}

En correspondencia metódica al análisis de discurso utilizado en este estudio, se examinaron los significados e implicaciones de las narrativas planteadas por los profesionales entrevistados. Según presentado anteriormente, las preguntas de investigación que guían el contenido de este artículo son: (1) ¿Cómo definen el trabajo social clínico quienes ejercen la profesión?; y (2) ¿Cuáles aspectos del ejercicio del trabajo social clínico son materia de debate en Puerto Rico? La primera observación pertinente que debemos establecer al atender estas preguntas, es que sus respuestas se entrelazan. Es decir, las verbalizaciones relacionadas a la definición del TSC se convierten en un tema de debate. A su vez, temas de debate, son considerados imprescindibles para definir el TSC. Debido a esta situación, se procede a discutir de forma entrelazada ambos tópicos, dando por resultado los alcances y debates del TSC, como eje principal del artículo.

Al repasar los distintos discursos recopilados en esta investigación, solo podemos establecer un aspecto de forma concluyente. Ese aspecto es que las visiones sobre el TSC en Puerto Rico son heterogéneas, lejos de definitivas. Es importante destacar la existencia de choques entre concepciones sobre el TSC, aún en profesionales con formaciones académicas similares como las personas que participaron de la investigación. Nótese que es común a los adentros de cualquier profesión, que haya debates en cuanto a marcos conceptuales, teóricos o de referencia sobre cómo desenvolverse o accionar en una disciplina. En este artículo se presenta que esta realidad está presente no solo en el TSC sino en el TS, como sucede en cualquier 
otra profesión. Sin embargo, no es igualmente común que no haya una percepción uniforme, desde los propios adentros de la profesión, como sucede al establecer los alcances del TSC.

En ocasiones, y tal vez llevados por el impulso discursivo de la visión holística del TS, puede haber propensión a defender la existencia y función del TSC, sin que se presenten cuestionamientos sobre su naturaleza. Tampoco se cuestiona la calidad de la formación recibida, o el contraste de la idealización sobre cuáles son las competencias del TSC versus la formación que realmente se ofrece y recibe para tal título. Nos circunscribimos a Garcés (2010), quien sostiene: "La especialización y la formación implican educarse en la autocrítica y en la heterocrítica, situarse en la posición permanente de aprender, de estar en proceso, de saber mirarse al espejo y evaluarse como parte del análisis de la situación" (p. 348).

Por otro lado, se hacen observaciones importantes en cuanto a la pertinencia e importancia de que se ofrezcan servicios de índole clínica desde el TS que pongan la mirada y acciones en las situaciones comunitarias, políticas y estructurales; o bien sea, sin perder de vista la justicia social con la cual nos comprometemos al formarnos como profesionales del TS. La finalidad terapéutica de intervenir con malestares emocionales implica la consideración de otros aspectos más allá de lo clínico (Ituarte, 2017). Si no, sería un servicio clínico sin identidad de TS.

Igualmente es relevante enfatizar dentro de la práctica clínica aquellos aspectos relacionados a las diversidades e interseccionalidades, con el fin de realizar intervenciones críticas y anti-opresivas en las que se reconozca la influencia de los factores antes mencionados, más allá de los llamados signos y síntomas identificados. Asimismo, accionar reconociendo que las patologías conductuales 0 cognitivas de una persona, deben ser consideradas e interpretadas como sintomatologías que responden a los contextos o circunstancias sociales. Por consiguiente, también deben interpretarse e intervenirse a nivel estructural. No obstante, las verbalizaciones obtenidas no enfatizan que la formación del TSC haga hincapié en tales miradas. Algo es mencionado al respecto cuando se describe al TSC, pero no así al discutir la formación. Si estos aspectos no son enfatizados en la formación de TSC, los argumentos que defienden la relación inherente entre el TSC y los factores comunitarios, así como de justicia social, pasan a ser más 
un discurso de lo deseable, que una descripción de la realidad.

Por estas circunstancias, la formación del TSC se torna en un elemento importante a considerar. Sobre este particular hay que mirar la tendencia de las universidades a ofrecer formaciones más rápidas y menos habilitantes. Características como la tendencia al "fast track", y la lenidad académica, afectan la formación del TSC al reducir las posibilidades de que trabajadores sociales en adiestramiento adquieran dominio real sobre epistemología y técnicas de intervención, entre otras áreas. Convendría explorar los contenidos curriculares de los programas de TSC para evidenciar cuánto énfasis se pone en las formas de intervención que, desde la consideración clínica, analicen e incorporen las circunstancias ambientales y estructurales que generan la sintomatología clínica. Sin embargo, como ilustraron las entrevistas, con frecuencia los marcos teóricos y de referencia para la intervención surgen eminentemente de la psicología sin que se plantee realmente una mirada y ejecución que sea particular y característica del TSC. Al respecto Reyes (2012), expone: "La gran mayoría de las veces pensamos que la intervención social como tal, está enraizada en conocimientos externos a nuestro propio hacer. Dicho en otras palabras, negamos que nuestro hacer tenga cimientos teóricos fundados desde nuestro saber-hacer disciplinar" (p. 21).

Es pertinente destacar que esta observación no atañe únicamente al TSC. EI TS, de forma general, ha estado siendo utilizado para el asistencialismo y para la implementación de políticas sociales que aparentan brindar 'ayuda' a la población. Pero, por su naturaleza, consiguen beneficiar al sistema capitalista mientras mantiene a la población en condiciones precarias (Montaño, 2000). Esto no descarta la intención noble y gestiones loables de nuestros colegas, pero, en esa función, queda poco espacio para estimular una acción social emancipadora. Entiéndase:

...llevar a cabo una praxis para que las cosas puedan ser de otra manera en la vida cotidiana de los sujetos sociales, mediante la cimentación de identidades, la resignificación del mundo de la vida, la formación de lazos sociales menos desiguales y más democráticos, la alzadura de ciudadanía y, de este modo, la materialización del derecho a tener derechos (Martínez \& Agüero, 2015, p. 16).

Esta reflexión, es sumamente necesaria al interior de toda la profesión en Puerto Rico. No se trata únicamente del TSC. Los 
señalamientos centrados en el TSC que se presentan en este escrito se deben a que esta especialidad es el eje de atención de la investigación realizada. Hay que señalar que según ABECSW (2019), los profesionales del TSC son el grupo más numeroso de proveedores de servicios de salud mental en los Estados Unidos. Aunque no hemos tenido acceso a datos que puedan constatar ese hecho en Puerto Rico, es una realidad que la oferta de las instituciones post-secundarias ha aumentado en lo que respecta al TSC. Centrar las observaciones en el TSC se debe, además, a que en este momento histórico, las tendencias neoliberales han encontrado una nueva forma de beneficiar al capitalismo teniendo por medio instrumental al TSC (Barreto et al., 2016). Tómese, por ejemplo, que en muchos escenarios las funciones desempeñadas por el TSC son regidas por empresas de seguro médico que buscan cumplir con un servicio que resulte conveniente a las finanzas de la entidad privada. Igualmente, debe analizarse si realmente en la mayoría de los escenarios del TSC es una práctica común la consideración de las circunstancias personales, comunitarias y estructurales o, en su lugar, el TSC es utilizado como una alternativa "más barata" de servicios clínicos que convienen al estado capitalista. Vale destacar, entre otras muchas consideraciones importantes, que el TSC suele ser desempeñado por un profesional con un grado de maestría. Mientras, otros profesionales clínicos requieren un grado doctoral para el ejercicio de su profesión resultando más costoso.

En este apartado, cabe también la consideración conflictiva de un TSC que se circunscribe al trabajo profesional con salud mental resolviendo sus intervenciones, primordialmente, en función de la psiquiatría o la psicología. Si bien se defiende la legitimidad del TSC con funciones y aportaciones particulares, es innegable la inherencia e influencia que tiene la disciplina de la psicología en la configuración del TSC en Puerto Rico. Cuando el marco referencial para las intervenciones profesionales se ciñe a concepciones de otra disciplina, se trastoca la propia identidad profesional y su evolución. Por ejemplo, hay que reflexionar sobre las implicaciones de un TSC que incorpore acríticamente la implementación del DSM, instrumento medular para la psicología clínica y la psiquiatría. Considérese que utilizar el DSM como un instrumento útil para el análisis y canalización de las intervenciones no es lo mismo que limitar las intervenciones al marco patologizante que el uso del Manual supone. Queda por ver cuánto de esa diferenciación realmente es desempeñada por el TSC. Asimismo, debe reflexionarse si en la intervención seconsideraal participante, llanamente, 
como portador de un trastorno, sin que hayan sido sopesadas las dimensiones sociales y estructurales que conducen a tales circunstancias. Es necesario destacar que, a diferencia de otras profesiones, el TS tiene por médula la justicia social y la defensa de los derechos humanos, así como el análisis y acciones ante los problemas socio-estructurales y sus implicaciones para individuos, familias, grupos y comunidades. Adherirse acríticamente a las visiones y procederes de otra profesión, transmuta la realización de dicha médula.

Este planteamiento no debe confundirse con la realidad objetiva de que el desarrollo del TS ha sido nutrido por otras áreas del saber, como: la educación, economía, sociología, política y la propia psicología. Lo que aquí se señala es que al permitir que una de estas fuentes disciplinares imbuya y sature el quehacer del TS, se desvirtúa su identidad y accionar. Similarmente, pero en un polo opuesto, sucede cuando se concibe un TS que únicamente postula desde el análisis de las situaciones político-económicas-estructurales desconsiderando los aspectos personales, microestructurales o la propia liberación de las personas. $\mathrm{Si}$ bien la primera confunde al TS con la disciplina de la psicología, la segunda le confunde con sociología, ciencias políticas u otra de orden similar.
Este aspecto también merece serias consideraciones y discusión, pero no es el eje de este artículo.

El debate entre la naturaleza política del TS y la dimensión de las intervenciones terapéuticas puede aparentar cierto impase de carácter dicótomo, pero no tiene que ser así. En el propio Código de Ética Profesional (CPTSPR, 2017) se define al TS como una profesión que "fundamenta su acción ético-política en la defensa y ampliación de los derechos humanos" (p.82) e igualmente establece que el TS se inserta en distintas áreas que incluyen las terapéuticas para incidir "en el fortalecimiento de la autonomía, el ejercicio de la ciudadanía y el bienestar integral del ser humano en todos sus contextos" (p.82). Uno de esos contextos es la salud, reconocida como un derecho humano (Rivera, 2018, p. 27). La Organización Mundial de la Salud (1948) establece que: "la salud es un estado de completo bienestar físico, mental y social, y no solamente la ausencia de afecciones o enfermedades" (p.1). Así pues, es necesario reconocer la atención a la Salud Mental como parte de estos derechos que deben atenderse íntegramente; y para lo cual, el TS tiene deber y competencias.

De la realización de este artículo proponemos dos recomendaciones específicas. La primera es trabajar 
una definición consensuada de lo que significa TSC en Puerto Rico. Aunque existen varias definiciones, por ejemplo: ABECSW (2019); Antipan y Reyes (2015); Garcés (2010); Huaiquiche \& Bastías (2016); Ituarte (2017); NASW (2019); es preciso reconocer que la realidad de la práctica en Puerto Rico, así como las reflexiones en torno a la misma, no suponen necesariamente que el TSC que se conceptúa en Puerto Rico, quede sujeto a alguna de ellas. Hacer el ejercicio de elaborar una definición del TSC en Puerto Rico, podría proveer de un espacio para diálogo e integración entre distintos sectores y profesionales del TSC con perspectivas diversas. Igualmente, se desprende de las expresiones de las personas entrevistadas en este estudio, la necesidad de generar modelos de intervención propios del TSC. Sin embargo, para realizar esa tarea es preciso que antes se esclarezca la identidad y alcances del TSC. Incluso, la propia denominación del TSC, debería estar sujeta a discusión. La literatura trata los términos Trabajo Social Terapéutico - Dimensión Terapéutica del Trabajo Social (Barreto et al., 2016; Rojas, 2011), distanciándose del carácter patológico del término 'clínico'. Nótese que dicho término proviene del latín 'clinǐcus', que significa 'propio del enfermo' (Real Academia Española, 2019). Lo anterior, entre otras consideraciones podrían ser sopesadas.
La segunda recomendación que planteamos es realizar un estudio que documente las prácticas reales que están llevando a cabo profesionales del TSC en diversos escenarios en Puerto Rico. Que se documenten los servicios que se ofrecen; las competencias que realmente descargan; y cuán afines son las funciones desempeñadas con las bases comunitarias y de justicia social. Esto permitiría hacer análisis basados en circunstancias concretas, más que en los discursos o percepciones de algunos pocos.

Definitivamente hay más recomendaciones por hacer, así como otras consideraciones a discutir sobre el tema tratado en este escrito. Hemos procurado integrar literatura local e internacional sobre el TSC que resulte relevante a las particularidades del tema tratado. Sin embargo, la información producida al respecto en PR resulta escasa. Confiamos que este trabajo contribuya al surgir de nuevas investigaciones que aporten a esta discusión. Ya se perfila una generación de profesionales que obtendrán grados doctorales en TSC. Habrá que observar las implicaciones que tendrá el aumento de profesionales con doctorado en TSC, en la medida que va deviniendo su inserción en escenarios de servicio.

Antes de concluir el escrito, debe señalarse que esta investigación no 
pone en duda las aportaciones que realizan muchos profesionales con práctica clínica. Lejos de poner en duda, reconocemos sus competencias profesionales, cualidades éticas y compromiso social. Más allá, y como es mencionado anteriormente, muchos planteamientos que aquí se ciernen no se circunscriben únicamente al TSC, sino que expresan una realidad del TS como profesión, independientemente de especialidades. Postulamos que debería reflexionarse activamente sobre la propia naturaleza y funciones de lo que implica el ejercicio del TS en todas sus áreas.

Para finalizar, reafirmamos que el propósito principal de este artículo ha sido abrir discusiones que permitan reflexionar y estimular diálogos. Proponemos como una práctica saludable, que cada cual se aventure a retar sus propias concepciones. No solo escuchar para refutar, sino escuchar para reflexionar y aceptar aquellas posturas que nuestra honestidad intelectual nos permita. Poseer la capacidad de pensamiento crítico no es solo problematizar ideas contrarias o posturas establecidas. También se trata de reconocer que las ideas, propias y ajenas, pueden evolucionar. Para transformar la realidad, es necesario comenzar por reconocerla.

\section{Agradecimiento}

Los investigadores agradecen la participación de los profesionales que accedieron a ser entrevistados en este estudio.

\section{Declaración de Conflicto de intereses}

Las personas autoras declararon que no hay conflictos de interés potenciales con respecto a la investigación, autoría o publicación en este artículo.

\section{Declaración sobre lenguaje inclusivo}

En esta investigación se utilizará, principalmente, el género gramatical masculino para referirse a diversas identidades humanas o colectividades (por ejemplo, trabajadores y trabajadores sociales; hombre, mujer, queer, intersexuales, por mencionar algunos) sin que esto suponga un lenguaje sexista y excluyente.

\section{Financiamiento}

Los autores informaron que este artículo es una publicación parcial de una investigación realizada con una aportación económica de la Vicerrectoría de Asuntos Académicos de la Universidad Ana G. Méndez, Recinto de Carolina, entonces Universidad del Este. 


\section{Referencias}

American Board of Examiners in Clinical Social Work [ABECSW] (2019). Clinical Social Work Described. Recuperado de: https://abecsw.org/clinicalsocial-work/clinical-social-workdescribed/

Antipan, I., \& Reyes, D. (2015). El trabajo social en salud mental: un trabajo social clínico. Recuperado de: https://www.researchgate. net/publication/328475212_El_ Trabajo_Social_en_Salud_Mental_ Un_Trabajo_Social_Clinico

Babbie, E., \& Rubin, A. (2011). Research methods for Social Work (7ed). USA: Brooks/Cole, Cengage Learning.

Barreto, E., Rosario, N., \& Sánchez, F. (2016). El derecho a la salud y la política social precarizada: desafíos de la dimensión terapéutica en el trabajo social. Voces desde el Trabajo Social, 4(1), 17-58.

Colegio de Profesionales del Trabajo Social de Puerto Rico [CPTSPR]. (2017). Código de ética profesional. San Juan: Autor.
Córdova, R. (2010). Trabajo

social clínico en Puerto Rico:

Construcción de la personalidad puertorriqueña. Puerto Rico: Action Printing.

Elçi, A., \& Devran, B. (2014). A Narrative Research Approach: The Experiences of Social Media Support in Higher Education. En: Zaphiris P., loannou A. (eds) Learning and Collaboration Technologies. Designing and Developing Novel Learning Experiences. Lecture Notes in Computer Science, 8523(1). Springer: Cham.

Ellard-Gray, A., Jeffrey, N., Choubak, M., \& Crann, S. (2015). Finding the Hidden Participant: Solutions for Recruiting Hidden, Hard-to-Reach, and Vulnerable Populations. International Journal of Qualitative Methods. Recuperado de: https:// journals.sagepub.com/doi/ full/10.1177/1609406915621420

Garcés, E. (2010) El Trabajo Social en Salud Mental. Cuadernos de Trabajo Social, 23(1), 333-352. Recuperado de: http://revistas. ucm.es/index.php/CUTS/article/ view/CUTS1010110333A/7489 
Hernández, R., Fernández, C., \& Baptista, P. (2014). Metodología de la Investigación. (6 $6^{\mathrm{a}}$. ed.). México: McGraw Hill.

Huaiquiche, T., \& Bastías, C. (2016). Trabajo social y práctica clínica individualizada-familiar en salud mental: una mirada analítica y sociohistórica. Revista Electrónica de Trabajo Social, 13(1), (33-50.

Ituarte, A. (Coord.). (2017). Prácticas del Trabajo Social Clínico. Valencia: Nau Libres.

Martínez, S., \& Agüero, J. (2015). La intervención social desde la perspectiva del trabajo social emancipador. Repositorio Institucional, 4(1), 9-16.

Mirabito, D. (2012). Educating a new generation of social workers: Challenges and skills needed for contemporary agency-based practice. Clinical Social Work Journal, 40(2), 245-254.

Montaño, C. (2000). La naturaleza del servicio social: un ensayo sobre su génesis, su especificidad y su reproducción. San Pablo: Editorial Cortez.
National Association of Social Work (NASW) (2019). Clinical Social Work. Recuperado de: https:// www.socialworkers.org/Practice/ Clinical-Social-Work

Organización Mundial de la Salud (1948). Constitución de la Organización Mundial de la Salud. Recuperado de https://www. who.int/governance/eb/who_ constitution_sp.pdf

Padgett, D. (2008). Qualitative Methods in Social Work Research (2 Ed.). USA: Sage

Real Academia Española [RAE]. (2019). Clínico. Edición Tricentenario. Recuperado de https://dle.rae.es/?id=9TNmHzU

Reyes, D. (noviembre, 2012). Trabajo social clínico, salud mental y psicoterapia: pasos hacia una práctica integral en los servicios de salud mental y psicosociales. Trabajo presentado en el Congreso Nacional de Estudiantes de Trabajo Social. Chile: Universidad Arturo Prat de lquique. 
Rivera, M. (2018). Derechos

humanos en la salud y trabajo

social en Puerto Rico: un ensayo

reflexivo para una propuesta de

acción. San Juan, Puerto Rico:

Publicaciones Puertorriqueñas.

Rojas, C. (2011). La dimensión terapéutica del Trabajo Social y la protección de los derechos humanos. Revista Costarricense de Trabajo Social (1, especial), 19-23.

Ryan, F., Coughlan, M., \& Cronin, P. (2009). Interviewing in qualitative research: the one-to-one interview. International Journal of Therapy and Rehabilitation, 16(6), 309-314.

Sayago, S. (2014). El análisis del discurso como técnica de investigación cualitativa y cuantitativa en las ciencias sociales. Cinta Moebio, 49(1), 1-10. doi:10.4067/S0717554X2014000100001 